\title{
The formation and disappearance of filament barbs observed by SDO
}

\author{
Leping Li and Jun Zhang
}

Key Laboratory of Solar Activity, National Astronomical Observatories, Chinese Academy of Sciences, Beijing 100012, China

email: lepingli@nao.cas.cn

\begin{abstract}
Employing six-day (August 16-21, 2010) SDO/AIA observations, we systematically investigate the formation and disappearance of 58 barbs of a northern $(\sim \mathrm{N} 60)$ polar crown filament. Three different ways of barb formation are discovered, including (1) the convergence of surrounding moving materials $(55.2 \%),(2)$ the flows of materials from the filament $(37.9 \%)$, and (3) the material injections from neighboring brightening regions $(6.9 \%)$. We also find three different types of barb disappearance, involving: (i) the bi-lateral movements (44.8\%), and (ii) the outflowing $(27.6 \%)$ of barb material resulting in the barb disappearance, as well as (iii) the barb disappearance associated with neighboring brightenings (27.6\%). We propose that barbs exchange materials with the filament, surrounding atmosphere, and nearby brightening regions, causing the barb formation and disappearance.
\end{abstract}

Keywords. Sun: filaments, Sun: prominences, Sun: evolution, Sun: corona

\section{Introduction}

Solar filaments are characterized by relatively cool and dense plasma (Babcock \& Babcock 1955, Schmieder et al. 2008). They typically have two linked substructures: the 'spine' and the 'barbs', between which the latter are made when some threads of the spine smoothly bend outwards (Lin 2008).

For the formation of the barbs, Wang (1999) presented that magnetic reconnection between the parasitic and the neighboring dominant polarities plays a key role. In contrast, Martens \& Zwaan (2001) suggested that the barbs arise as a result of failed cancelation. Heinzel \& Anzer (2001) suggested that barbs could be formed by the evaporationcondensation process if they are composed of vertically aligned dips. Aulanier et al. (1998) and Mackay \& van Ballegooijen (2009) also proposed that the barbs are cool matter suspended in local dips.

Most of the studies about barbs are based on $\mathrm{H} \alpha$ observations. After the launch of the Solar Dynamics Observatory (SDO), its high-quality observations provide us the opportunity to study the barbs in extreme ultraviolet (EUV) wavelengths. In this work, we present our systematic study of the formation and disappearance of barbs on a polar crown filament in six days. The observations are described in Section 2. We present the results and conclusions in Section 3, as well as the discussion, in Section 4.

\section{Observations}

The SDO/AIA is designed to acquire images of the solar atmosphere in a series of wavelength bands. In this study, we use the AIA $171 \AA$ and $304 \AA$ data to investigate the evolution of barbs. The time cadence and spatial resolution of the AIA images are $12 \mathrm{~s}$ and $1.2^{\prime \prime}$, respectively. We also employ the HMI line of sight magnetograms, with spatial resolution of $1.0^{\prime \prime}$ and time cadence of $45 \mathrm{~s}$, to study the evolution of magnetic fields surrounding the barbs. 


\section{Results and Conclusions}

From August 16 to 21, 2010, a northern (N50 N70) polar crown filament was observed by SDO. Using six-day observations, we identified 69 barbs, and chose 58 of them, which formed away from the western solar limb $(\leqslant \mathrm{W} 60)$, as our sample to study the barb formation and disappearance in detail.

\subsection{The formation of filament barbs}

Three different types of barb formation are detected (Li \& Zhang 2013), involving: (1) the convergence of moving materials, (2) the flows of material from the filament main body, and (3) the material injections from neighboring brightening regions.

$32(55.2 \%)$ barbs display the first type of barb formation. An example on August 16, 2010, is shown in the upper panels of Fig. 1. A barb is presented at 12:44 UT in Fig. 1a3. We overlay the barb on Fig. 1a1 as red contours, and notice that there was no barb at 11:36 UT in and surrounding the contour area. However, several materials moved around. Sometimes, some of the materials, denoted by three red arrows in Fig. 1a2 with heads showing the moving directions, converged together. Finally, a new barb formed. In short, the ambient moving materials converge together forming a new barb.

$22(37.9 \%)$ barbs manifest the second type of barb formation. We display an example on August 16, 2010, in the middle panels of Fig. 1. Figure 1b3 illustrates a barb at 9:23 UT. We overly the barb as black contours on Fig. 1b1, and find that there was no barb existed at 9:07 UT. However, to the northwest, some small-scale materials flowing along the spine were detected. From 9:13 UT, the horizontal flows transformed into southward

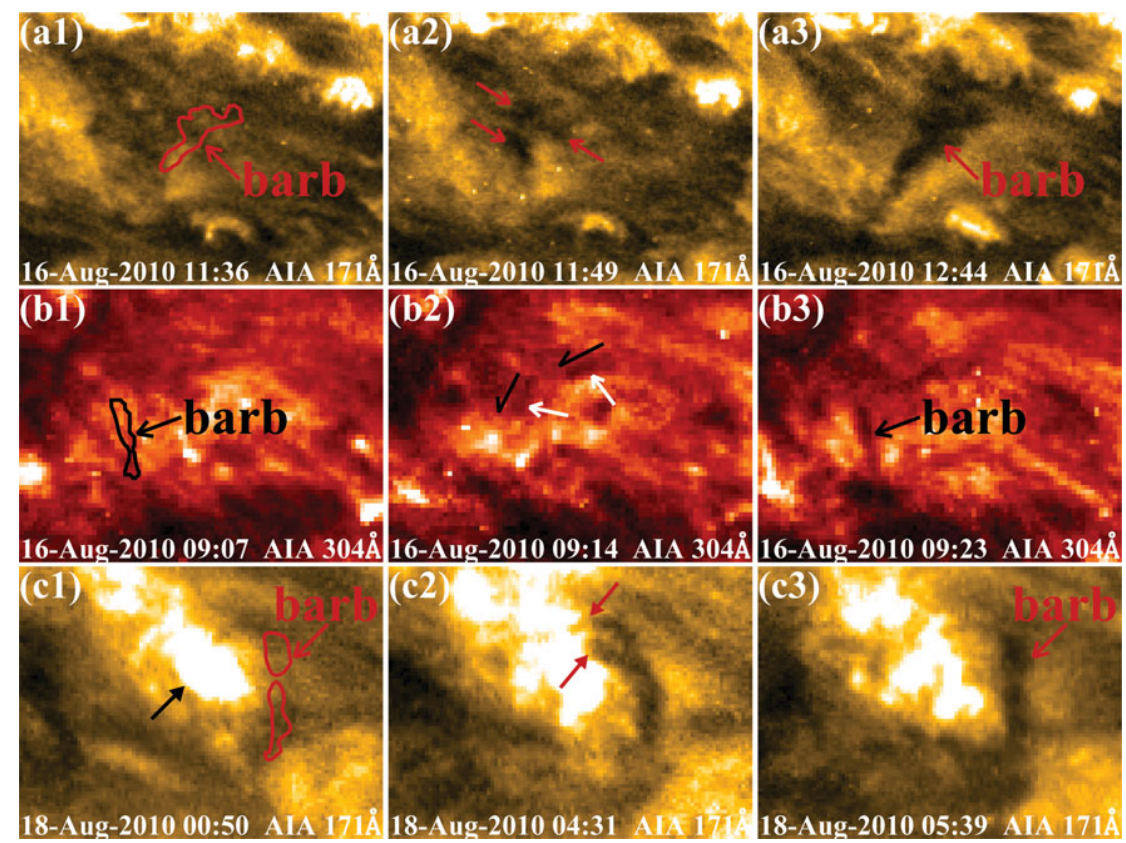

Figure 1. A series of SDO/AIA $171 \AA$ (a1-a3), $304 \AA$ (b1-b3), and $171 \AA$ (c1-c3) images displaying the formation of a filament barb on August 16, 16, and 18, 2010, respectively. The contours in (a1), (b1), and (c1) separately enclose the barb in (a3), (b3), and (c3). Three red arrows in (a2) and two white arrows in (b2) denote the moving materials, and two black arrows in (b2), the moving directions. A black arrow in (c1) indicates a brightening region, and two red arrows in (c2), the connections between the brightening regions and the barb. The field of view (FOV) of (a1-a3), (b1-b3), and (c1-c3) is $96^{\prime \prime} \times 72^{\prime \prime}, 48^{\prime \prime} \times 36^{\prime \prime}$, and $80^{\prime \prime} \times 45^{\prime \prime}$, respectively. 
flows (see the black arrows in Fig. 1b2). Finally, a new barb formed. In a word, the transition of the flows along the spine into downward flows forms a new barb.

In $4(6.9 \%)$ cases of our sample, another type of barb formation is noted. An example on August 18, 2010, is displayed in the lower panels of Fig. 1. A barb is indicated in Fig. $1 \mathrm{c} 3$ at 5:39 UT. We overlay the barb as red contours on Fig. 1c1, and notice that there was no barb at 0:50 UT. Nevertheless, several neighboring brightening regions existed to the east of the contour regions. From 0:25 UT, the brightening regions began to inject small-scale materials into the contour area to form a new barb. Two connections between the brightening regions and the new formed barb appeared, labeled by two red arrows in Fig. 1c2. Finally, these connections disappeared, and the barb formed. In brief, the materials injected from the neighboring brightening regions form a new barb.

\subsection{The disappearance of filament barbs}

Barbs are seen to disappear in three different ways (Li \& Zhang 2013), involving: (i) the barb moves bi-laterally and disappears into the surrounding atmosphere, and (ii) the outflowing of barb materials to the chromosphere results in the barb disappearance, as well as (iii) the barb disappearance is associated with neighboring brightenings.

In our sample, $26(44.8 \%)$ barbs illustrate the first way to disappear. An example on August 18, 2010, is displayed in the upper panels of Fig. 2. Figure 2a1 shows a barb at 8:10 UT. Thereafter, the barb moved bi-laterally (see the green arrows in Fig. 2a2), and disappeared rapidly into the atmosphere. We overlay the barb as red contour on Fig. 2a3, and find that the barb completely disappeared at 12:39 UT. In brief, the bi-lateral movements of the barb lead to the barb disappearance.

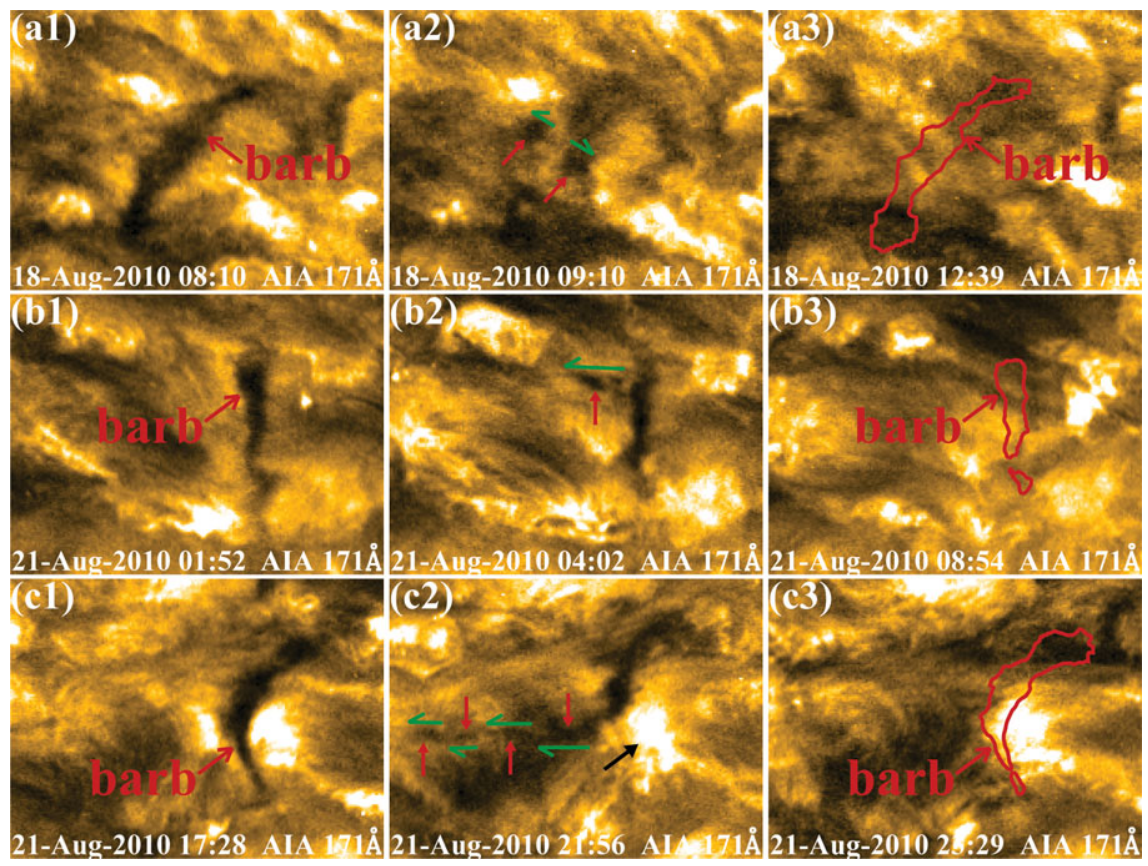

Figure 2. A series of SDO/AIA $171 \AA$ images separately showing the disappearance of a filament barb on August 18 (a1-a3), 21 (b1-b3), and 21 (c1-c3), 2010. The red contours in (a3), (b3), and (c3) enclose the barb in (a1), (b1), and (c1), respectively. The red arrows in (a2), (b2), and (c2) separately mark the moving materials, and green arrows, the moving directions. A black arrow in (c2) denotes a brightening region. The FOV of (a1-a3), (b1-b3), and (c1-c3) is $96^{\prime \prime} \times 72^{\prime \prime}$, $120^{\prime \prime} \times 90^{\prime \prime}, 120^{\prime \prime} \times 90^{\prime \prime}$, respectively. 
$16(27.6 \%)$ barbs display the second way to disappear. We demonstrate an example on August 21, 2010, in the middle panels of Fig. 2. A barb is presented at 1:52 UT in Fig. 2b1. From 2:43 UT, the barb materials began to outflow toward east, as denoted by a green arrow in Fig. 2b2. Consequently, the barb materials became less and less, and the barb disappeared. The red contours in Fig. $2 \mathrm{~b} 3$ enclose the barb in Fig. 2b1. There was no barb existed at 8:54 UT in the contour area. In short, the outflowing of the barb material leads to the barb disappearance.

$16(27.6 \%)$ barbs show the third type of barb disappearance. An example on August 21, 2010, is demonstrated in the lower panels of Fig. 2. A barb is marked at 17:28 UT in Fig. 2c1. We overlay the barb as red contours on Fig. 2c3, and note that the barb completely disappeared at 23:29 UT. During the disappearance process, a brightening region, indicated by a black arrow in Fig. 2c2, was detected to the west of the barb. The barb materials (denoted by red arrows in Fig. 2c2) moved toward the east one by one (see the green arrows in Fig. 2c2). In a word, the barb disappearance is associated with the nearby small-scale solar brightening.

\section{Discussion}

About the barb formation, two aspects are considered: the formation of (1) the barb magnetic structures, supporting the material, and (2) the barb material. We investigate the evolution of magnetic fields, and find that magnetic emergences may form the barb magnetic structures. About the origination of the barb material, three different types of formation are found, and the third type seems to be consistent with the injection models (Wang 1999) of the filament material.

Similar to the formation, two aspects are also regarded during the barb disappearance, including: the disappearance of (1) the barb magnetic structures, and (2) the barb material. After investigation of magnetic field evolutions, we find that the magnetic field motions, emergences, cancelations, and disappearances may deform/destroy the barb magnetic structures.

In a word, the evolution of the photospheric magnetic fields may be the reason for the formation and disappearance of the barb magnetic structures. Moreover, the barbs exchange materials with the surrounding atmosphere, the filament spine, and the neighboring small-scale brightening regions, caused by the magnetic cancelations, can lead to the formation and disappearance of the barb material.

\section{Acknowledgements}

The work is supported by the National Natural Science Foundations of China (G11003026, 11025315, 11303050, 11221063, 41074123, 11003024), the National Basic Research Program of China under grant G2011CB811403, and the CAS KJCX2-EW-T07.

\section{References}

Aulanier, G., Démoulin, P., van Driel-Gesztelyi, L., et al. 1998, Astron. Astrophys., 335, 309

Babcock, H. W. \& Babcock, H. D. 1955, Astrophys. J., 121, 349

Heinzel, P. \& Anzer, U. 2001, Astron. Astrophys., 375, 1090

Li, L. P. \& Zhang, J. 2013, Solar Phys., 287, 147

Lin, Y. 2011, Space Sci. Rev., 158, 237

Mackay, D. \& van Ballegooijen, A. 2009, Solar Phys., 260, 321

Martens, P. C. \& Zwaan, C. 2001, Astrphys. J., 538, 872

Schmieder, B., Bommier, V., Kitai, T., et al. 2008, Solar Phys., 247, 321

Wang, Y. M. 1999, Astrophys. J. Lett., 520, L71 